\title{
Engineering of acetyl-CoA metabolism for the improved production of polyhydroxybutyrate in Saccharomyces cerevisiae
}

\author{
Kanokarn Kocharin, Yun Chen, Verena Siewers and Jens Nielsen*
}

\begin{abstract}
Through metabolic engineering microorganisms can be engineered to produce new products and further produce these with higher yield and productivities. Here, we expressed the bacterial polyhydroxybutyrate (PHB) pathway in the yeast Saccharomyces cerevisiae and we further evaluated the effect of engineering the formation of acetyl coenzyme A (acetyl-CoA), an intermediate of the central carbon metabolism and precursor of the PHB pathway, on heterologous PHB production by yeast. We engineered the acetyl-CoA metabolism by co-transformation of a plasmid containing genes for native $S$. cerevisiae alcohol dehydrogenase $(A D H 2)$, acetaldehyde dehydrogenase (ALD6), acetyl-COA acetyltransferase (ERG10) and a Salmonella enterica acetyl-CoA synthetase variant (acs ${ }^{\text {L641P }}$, resulting in acetoacetyl-CoA overproduction, together with a plasmid containing the PHB pathway genes coding for acetyl-CoA acetyltransferase (phaA), NADPH-linked acetoacetyl-CoA reductase (phaB) and poly(3-hydroxybutyrate) polymerase (phaC) from Ralstonia eutropha H16. Introduction of the acetyl-CoA plasmid together with the PHB plasmid, improved the productivity of PHB more than 16 times compared to the reference strain used in this study, as well as it reduced the specific product formation of side products.
\end{abstract}

Keywords: Polyhydroxybutyrate, Acetyl coenzyme A, Saccharomyces cerevisiae, Pathway engineering

\section{Introduction}

Poly-(R)-3-hydroxybutyrate (PHB) is the most common type of polyhydroxyalkanoates (PHAs) synthesized and accumulated by microorganisms like Ralstonia eutropha (also known as Cupriavidus necator, Wautersia eutropha, Alcaligenes eutrophus), Bacillus megaterium or Pseudomonas sp. as carbon and energy storage material in response to conditions of physiological stress (Steinbuchel et al. 1993). Biodegradable PHB is a linear polyester consisting solely of the stereospecific monomer, (R)-3-hydroxybutyric acid. It belongs to the group of short chain length PHAs consisting of $\mathrm{C}_{3}-\mathrm{C}_{5}$ hydroxyacid monomers. (Hankermeyer and Tjeerdema 1999; Melchiors et al. 1994). The biosynthesis pathway of PHB involves three enzymes and their sequential reactions (Breuer et al. 2002; Carlson et al. 2002; Steinbüchel 2001; Steinbüchel and Hein 2001). The first enzyme of the pathway is acetylCoA C-acetyltransferase [EC 2.3.1.9], encoded by phaA,

\footnotetext{
* Correspondence: nielsenj@chalmers.se

Department of Chemical and Biological Engineering, Chalmers University of Technology, Kemivägen 10, SE-412 96 Göteborg, Sweden
}

which catalyzes the condensation of two acetyl-CoA molecules to form acetoacetyl-CoA (Peoples and Sinskey 1989b). The next step is the reduction of acetoacetylCoA to (R)-3-hydroxybutyryl-CoA, which is catalyzed by NADPH-dependent acetoacetyl-CoA reductase [EC 1.1.1.36] encoded by phaB (Peoples and Sinskey 1989b). Finally, PHA synthase [EC 2.3.1.-] encoded by phaC, catalyzes the polymerization of (R)-3-hydroxybutyryl-CoA monomers to PHB (Peoples and Sinskey 1989a).

The natural PHB producers like $R$. eutropha, Bacillus megaterium or Pseudomonas sp. are known to produce and accumulate $\mathrm{PHB}$ as a storage compound in response to nutrient imbalance caused by growth under conditions of carbon source excess but limitation in other essential nutrients (Steinbüchel and Hein 2001; Trotsenko and Belova 2000). Instead of employing the natural PHB producers, which can depolymerize PHB and use it as a secondary energy source, metabolic engineering can be used to transfer the PHB biosynthetic pathway to alternative hosts that may have advantage over the natural PHB producers, in particular the lack of enzymes for PHB 
depolymerization (Uchino and Saito 2006). Furthermore, by transferring to alternative hosts one may take advantage of a range of technologies developed for general platform cell factories like Escherichia coli and Saccharomyces cerevisiae. Thus, there have been a range of studies where PHB production has been evaluated in E. coli and further metabolic engineering has been carried out with the objective to improve the productivity. In a metabolic and kinetic study, a recombinant $E$. coli strain producing PHB was examined and compared to the native $\mathrm{PHB}$ producer, $R$. eutropha, and this study revealed that the PHB flux was highly sensitive to the acetyl-CoA/CoA ratio, the total acetyl-CoA plus CoA concentration and $\mathrm{pH}$ (van Wegen et al. 2001). In recombinant $E$. coli, a mutation in $\operatorname{arc} A$ encoding a protein that regulates aerobic respiration under microaerobic conditions resulted in higher amounts of PHB accumulated in the cell (Nikel et al. 2006). Lowagitation conditions had a positive effect on PHB synthesis from glycerol in recombinant $E$. coli carrying the phaCAB operon and phaP encoding a granule-associated protein (phasin) (de Almeida et al. 2010). Several studies also attempted to synthesize $\mathrm{PHB}$ in plants according to the aim to produce high amounts of PHB at lower costs compared to microbial fermentation, particularly in plant plastids where the biosynthesis of fatty acids from acetylCoA occurs (Bohmert et al. 2000; Nawrath et al. 1994; Petrasovits et al. 2012). However, the growth of some of these transgenic plants was inhibited, possibly due to the metabolic burden of PHB synthesis (Poirier et al. 1992).

The benefits of using $\mathrm{S}$. cerevisiae as a model for producing $\mathrm{PHB}$ is that the molecular machinery of $S$. cerevisiae is well studied and as the most widely used eukaryal microorganism for industrial production of fuels and chemicals it is an attractive cell factory platform. Furthermore, its genome has been very well characterized and genome scale metabolic models have been reconstructed (Goffeau 2000; Nookaew et al. 2008). For these reasons, several attempts have been made to evaluate $S$. cerevisiae as a cell factory for PHB production (Breuer et al. 2002; Dimster-Denk and Rine 1996; Leaf et al. 1996; Marchesini et al. 2003; Zhang et al. 2006). Synthesis of PHB in S. cerevisiae has initially been demonstrated by expressing only bacterial polyhydroxybutyrate synthase (Leaf et al. 1996). This PHB synthesis approach is successful because of the activity of native thiolase and reductase enzymes involved in the synthesis of D-3-hydroxybutyryl-CoA in S. cerevisiae. However, the yield obtained in this study was very low when compared with the expression of all three genes of the PHB biosynthesis pathway (Breuer et al. 2002; Carlson et al. 2002; Carlson and Srienc 2006). Thiolase enzymes in $S$. cerevisiae exist and function in three different compartments, in mitochondria and peroxisomes for fatty acid $\beta$-oxidation and in the cytoplasm for the mevalonate pathway (Hiser et al. 1994). However, only the cytoplasmic thiolase participates in PHB biosynthesis. Many approaches have been followed to improve the production of PHB in yeast. Srienc and co-workers performed elementary mode analysis of a $S$. cerevisiae containing the PHB synthesis pathway in order to identify new metabolic engineering targets (Carlson et al. 2002). The analysis suggested that the introduction of the ATP citrate-lyase reaction and the transhydrogenase reaction can improve the theoretical PHB carbon yield (Carlson et al. 2002). AcetylCoA serves as the precursor for the PHB biosynthesis pathway and increasing the availability of acetyl-CoA was proposed to improve PHB production (Carlson and Srienc 2006; Suzuki et al. 2002). However, using enzyme inhibitors to reduce its consumption by other pathways or feeding of the substrate during cultivation would result in increasing production costs and may not be feasible for industrial applications. Here, we demonstrated metabolic pathway engineering by co-transformation of a plasmid containing the PHB biosynthesis pathway and an acetylcoenzyme A (acetyl-CoA) boost plasmid designated to improve the availability of cytoplasmic acetyl-CoA and hereby improve the productivity of PHB in S. cerevisiae.

\section{Materials and methods}

\section{Strains, media, and culture conditions}

Plasmids were maintained and propagated in E. coli DH5 $\alpha$. The preparation of competent E. coli cells and their transformation were performed according to standard protocols (Sambrook and Russell 2006). Lysogeny broth (LB) medium was used for routine culturing of $E$. coli (Bertani 1951) and $80 \mathrm{mg} \mathrm{L}^{-1}$ ampicillin was added to LB when needed. S. cerevisiae strain CEN.PK113-11C (MATa SUC2 MAL2-8 ${ }^{\mathrm{C}}$ ura3-52 his3- $\Delta 1$; provided by P. Kötter, Frankfurt, Germany) was used as the background strain for evaluation of the polyhydroxybutyrate pathway. Plasmid containing yeast strains were selected on synthetic dextrose (SD) medium, prepared with $6.7 \mathrm{~g} \mathrm{~L}^{-1}$ yeast nitrogen base without amino acids (YNBAA) (Formedium, Hunstanton, UK) and $20 \mathrm{~g} \mathrm{~L}^{-1}$ glucose with complete supplement mixture (CSM) lacking uracil and/or histidine (Formedium) where appropriate.

\section{Plasmid construction and yeast transformation}

The detailed construction of pIYC04 as a background plasmid and pIYC08 as acetyl-CoA boost plasmid is described by Chen et al. (Chen et al. 2012). The primers used for plasmid construction are listed in Table 1. The PHB biosynthesis pathway was introduced into CEN.PK 113-11C by using another multi-copy plasmid based on pSP-GM2 containing a $\mathrm{P}_{T E F 1}-\mathrm{P}_{P G K 1}$ bidirectional promoter (Partow et al. 2010). The PHB biosynthesis pathway genes $p h a A$, phaB and phaC were synthesized based on the genes from $R$. eutropha H16 and codon optimized for expression in $S$. cerevisiae by DNA 2.0 
Table 1 Oligonucleotides used in this study

\begin{tabular}{ll}
\hline No. & Sequence $\left(\mathbf{5}^{\prime} \mathbf{~} \mathbf{3}^{\mathbf{3}}\right)$ \\
\hline 1 & TACAATTGCTATTATTATCCTGCTCAGTGGTACTT \\
2 & TCCAATTGTCAGTGAGCGAGGAAGCGGAAGAG \\
3 & TTCGTTCTCCTTCTGTTCGGAGTTAC \\
4 & GGAACAGGAGTATTGCCTTTCAAGTAGTTATC \\
\hline Restriction sites are underlined.
\end{tabular}

(Menlo Park, CA, USA). PhaA was cloned into pSP-GM2 into the SpeI/SacI sites between the PGK1 promoter and the $A D H 1$ terminator. Then, PhaB was cloned into the BamHI/SalI sites between the TEF1 promoter and the CYC1 terminator of the same vector to yield PSP-GM2phaAB. PhaC was cloned into the MCS of pSP-GM2 vector the TEF1 promoter and the CYC1 terminator. The fragment of phaC together with the TEF1 promoter and the CYC1 terminator was amplified using primer 1 and 2 and ligated into pSP-GM2-phaAB using the MfeI restriction site resulting in $\mathrm{pKK} 01$. The direction of phaC insertion was confirmed by colony PCR with primer 3 and 4 . Yeast transformation was performed by using the lithium acetate/single-stranded carrier DNA/ polyethylene glycol method (Gietz and Woods 2002). Strain SCKK005 was constructed by transforming plasmids pKK01 and pIYC04 into strain CEN.PK113-11C. Plasmids pKK01 and pIYC08 were co-transformed into strain CEN.PK113-11C for the construction of SCKK006. Strain SCKK009 and SCKK010 were constructed by co-transformation of plasmids pKK01 and pIYC08 into SIYC32 and SCIYC33, respectively. The construction of the cit2 $\Delta$ strain (SCIYC32) and the $m l s 1 \Delta$ strain (SCIYC33) are described by Chen et al. (Chen et al. 2012). Strains used in this study are summarized in Table 2. The metabolic pathway and plasmid maps are illustrated in Figure 1.

\section{Shake flask cultivation}

The pre-cultures for shake flask cultivations were prepared by inoculation of $5 \mathrm{~mL}$ modified minimal medium in a $14 \mathrm{~mL}$ culture tube and grown at $30^{\circ} \mathrm{C}$ and 180 . The modified minimal medium for shake flask cultivations was prepared as follows: $5 \mathrm{~g} \cdot \mathrm{L}^{-1}\left(\mathrm{NH}_{4}\right)_{2} \mathrm{SO}_{4} ; 3 \mathrm{~g} \cdot \mathrm{L}^{-1}$ $\mathrm{KH}_{2} \mathrm{PO}_{4} ; 0.5 \mathrm{~g} \cdot \mathrm{L}^{-1} \mathrm{MgSO}_{4} \cdot 7 \mathrm{H}_{2} \mathrm{O} ; 1 \mathrm{~mL} \cdot \mathrm{L}^{-1}$ of trace metal solution and vitamin solution (see below) with an initial $\mathrm{pH}$ of 6.5. $45 \mathrm{~mL}$ of defined minimal medium in a $100 \mathrm{~mL}$ unbaffled flask were inoculated with an amount of pre-culture that resulted in a final optical density of 0.02 at $600 \mathrm{~nm}\left(\mathrm{OD}_{600}\right)$. The culture was grown at $30^{\circ} \mathrm{C}$ with $180 \mathrm{rpm}$ in an orbital shaking incubator and samples were taken at 40, 80 and $120 \mathrm{~h}$ to determine PHB production.

\section{Bioreactor cultivation}

$\mathrm{PHB}$ production was evaluated in defined minimal media with the following composition: $5 \mathrm{~g} \cdot \mathrm{L}^{-1}\left(\mathrm{NH}_{4}\right)_{2} \mathrm{SO}_{4}$; $3 \mathrm{~g} \mathrm{~L}^{-1} \mathrm{KH}_{2} \mathrm{PO}_{4} ; 0.5 \mathrm{~g} \mathrm{~L}^{-1} \mathrm{MgSO}_{4} \cdot 7 \mathrm{H}_{2} \mathrm{O} ; 1 \mathrm{~mL} \mathrm{~L}^{-1}$ trace metal solution ( $\mathrm{pH}$ 4.0: $15.0 \mathrm{~g} \mathrm{~L}^{-1}$ EDTA (sodium salt); $0.45 \mathrm{~g} \mathrm{~L}^{-1} \mathrm{ZnSO}_{4} \cdot 7 \mathrm{H}_{2} \mathrm{O} ; 1 \mathrm{~g} \mathrm{~L}^{-1} \mathrm{MnCl}_{2} \cdot 2 \mathrm{H}_{2} \mathrm{O} ; 0.3 \mathrm{~g} \mathrm{~L}^{-1}$ $\mathrm{CoCl}_{2} \cdot 6 \mathrm{H}_{2} \mathrm{O} ; \quad 0.3 \quad \mathrm{~g}^{-1} \mathrm{CuSO}_{4} \cdot 5 \mathrm{H}_{2} \mathrm{O} ; \quad 0.4 \mathrm{~g} \mathrm{~L}^{-1}$ $\mathrm{Na}_{2} \mathrm{MoO}_{4} \cdot 2 \mathrm{H}_{2} \mathrm{O} ; 0.45$ g L L $\mathrm{CaCl}_{2} \cdot 2 \mathrm{H}_{2} \mathrm{O} ; 0.3 \mathrm{~g} \mathrm{~L}^{-1}$ $\mathrm{FeSO}_{4} \cdot 7 \mathrm{H}_{2} \mathrm{O} ; 0.1 \mathrm{~g} \mathrm{~L}^{-1} \mathrm{H}_{3} \mathrm{BO}_{3}$, and $\left.0.10 \mathrm{~g} \mathrm{~L}^{-1} \mathrm{KI}\right)$. The $\mathrm{pH}$ was adjusted to 5 by adding $2 \mathrm{M} \mathrm{KOH}$ and autoclaved separately from the carbon source solution. Glucose was added at a concentration of $20 \mathrm{~g} \mathrm{~L}^{-1}$. Vitamin solution ( $\mathrm{pH}$ 6.5: $0.05 \mathrm{~g} \mathrm{~L}^{-1}$ biotin; $0.2 \mathrm{~g} \mathrm{~L}^{-1} \rho$-amino benzoic acid; $1 \mathrm{~g} \mathrm{~L}^{-1}$ nicotinic acid; $1 \mathrm{~g} \mathrm{~L}^{-1}$ Ca-pantothenate; $1 \mathrm{~g} \mathrm{~L}^{-1}$ pyridoxine- $\mathrm{HCl} ; 1 \mathrm{~g} \mathrm{~L}^{-1}$ thiamine- $\mathrm{HCl}$ and $25 \mathrm{~g} \mathrm{~L}^{-1}$ myo-inositol) was filter sterilized and aseptically added to the medium after autoclaving at the concentration of $1 \mathrm{~mL} \mathrm{~L}^{-1}$. Batch cultivations were carried out in $1.2 \mathrm{~L}$ bioreactors. The pre-cultures were prepared using the same culture conditions as for shake flask cultivation. $700 \mathrm{~mL}$ of defined minimal medium were inoculated with an amount of pre-culture that resulted in a final $\mathrm{OD}_{600}$ of 0.02 . The temperature was kept at $30^{\circ} \mathrm{C}$ and the $\mathrm{pH}$ was adjusted to $5.00 \pm 0.05$ using $2 \mathrm{M} \mathrm{KOH}$.

\section{Analytical methods}

Culture samples of $10 \mathrm{~mL}$ volume were centrifuged at $5,311 \times \mathrm{g}$ and $4^{\circ} \mathrm{C}$ for $5 \mathrm{~min}$ and the pellets were washed once with distilled water and centrifuged at $14,000 \times \mathrm{g}$

Table 2 Yeast strains and plasmids used in this study

\begin{tabular}{|c|c|c|c|}
\hline Strain & Genotype or relevant feature(s) & Plasmid & Source \\
\hline CEN.PK 113-11C & MATa SUC2 MAL2-8c ura3-52 his3- $\Delta 1$ & - & P. Kötter ${ }^{a}-$ \\
\hline SCKK005 & MATa SUC2 MAL2-8c ura3-52 his3- $\Delta 1$ & plYC04/pKK01 & This study \\
\hline SCKKO06 & MATa SUC2 MAL2-8c ura3-52 his3- $\Delta 1$ & plYC08/pKK01 & This study \\
\hline SCIYC32 & MATa SUC2 MAL2-8c ura3-52 his3- $\Delta 1$ cit2 $\Delta$ & - & (Chen et al. 2012) \\
\hline SCIYC33 & MATa SUC2 MAL2-8c ura3-52 his3- $\Delta 1 \mathrm{~m} / \mathrm{s} 1 \Delta$ & - & (Chen et al. 2012) \\
\hline SCKKOO9 & MATa SUC2 MAL2-8 $8^{\mathrm{C}}$ ura3-52 his3- $\Delta 1$ cit2 $\Delta$ & plYC08/pKK01 & This study \\
\hline SCKK010 & MATa SUC2 MAL2-8c ura3-52 his3- $\Delta 1 \mathrm{~m} / \mathrm{s} 1 \Delta$ & plYC08/pKK01 & This study \\
\hline
\end{tabular}

${ }^{a}$ Institute of Microbiology, J.W. Goethe Universität, Frankfurt, Germany. 


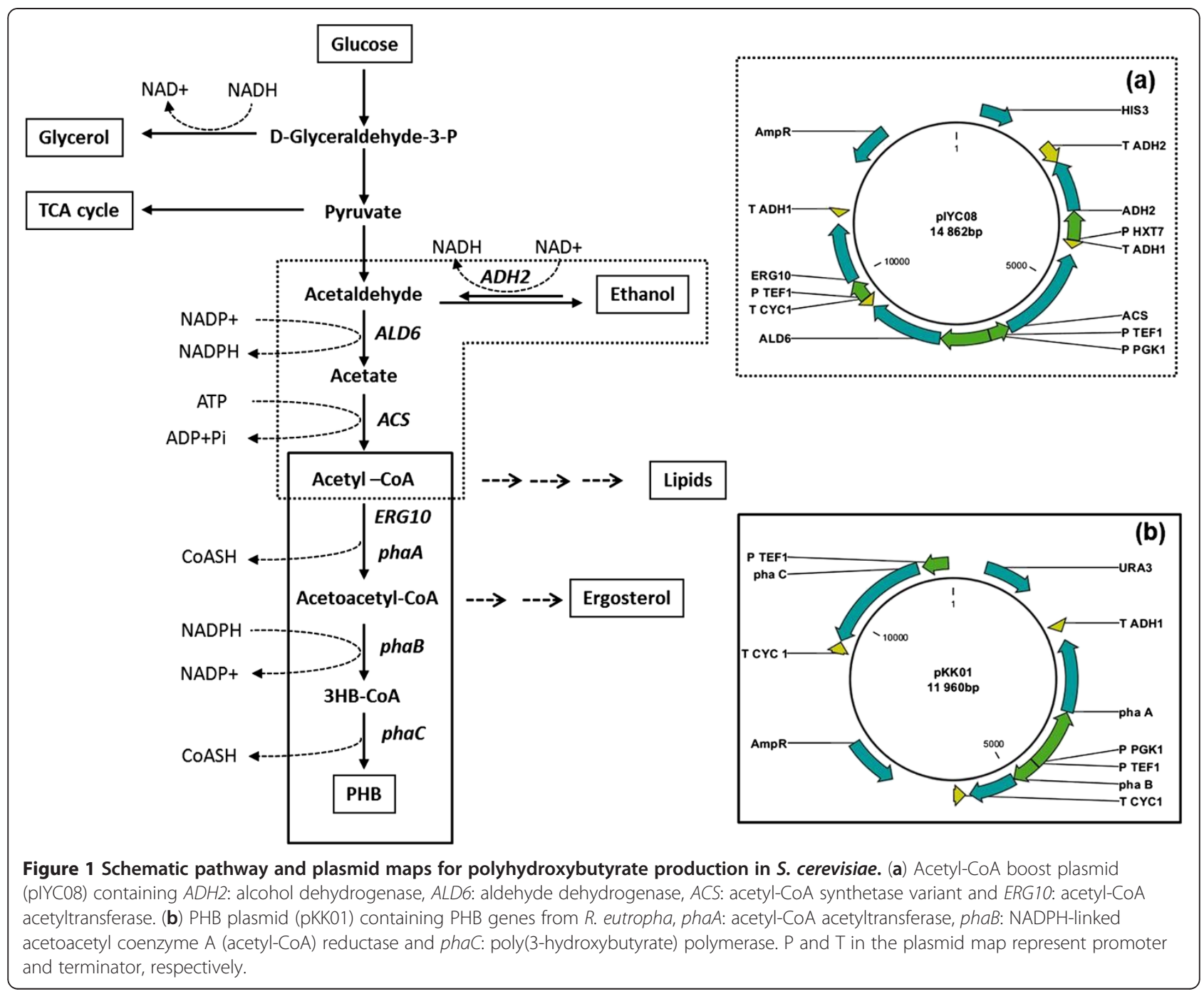

for $1 \mathrm{~min}$. To lyophilize the biomass, the recovered cell pellet was immediately frozen by immersion in liquid nitrogen followed by lyophilization under vacuum (Christ Alpha 2-4 LSC, Shropshire, UK). The cell dry weight was determined and the pellet kept at $4{ }^{\circ} \mathrm{C}$ for further analysis.

Metabolites including glucose, ethanol, glycerol, and acetate were quantified in the culture supernatant using an Ultimate 3000 HPLC (Dionex, Sunnyvale, CA, USA) equipped with an Aminex HPX $87 \mathrm{H}$ ion exclusion column (300 $\mathrm{mm} \times 7.8 \mathrm{~mm}$, Bio-Rad Laboratories, Hercules, CA, USA) which was operated at $45^{\circ} \mathrm{C}$ and a flow rate of $0.6 \mathrm{~mL} \mathrm{~min}{ }^{-1}$ of $5 \mathrm{mM} \mathrm{H}_{2} \mathrm{SO}_{4}$ using a refractive index detector and UV detector for analysis of sugars and organic acids, respectively.

PHB was analyzed as described previously (Karr et al. 1983; Tyo et al. 2006). 10-20 mg of dried cells were weighed and boiled in $1 \mathrm{~mL}$ of concentrated sulfuric acid for $60 \mathrm{~min}$ and then diluted with $4 \mathrm{~mL}$ of $14 \mathrm{mM}$
$\mathrm{H}_{2} \mathrm{SO}_{4}$. Samples were centrifuged ( $15 \mathrm{~min}, 16,000 \times \mathrm{g}$ ) to remove cell debris, and the supernatant was analyzed using an Ultimate 3000 HPLC (Dionex) equipped with an Aminex HPX-87 $\mathrm{H}$ ion exclusion column $(300 \times 7.8 \mathrm{~mm}$; Bio-Rad Laboratories $)$ and UV detector. Commercially available PHB (Sigma-Aldrich, St. Louis, $\mathrm{MO}$ ), processed in parallel with the samples, was used as a standard. The HPLC was operated at $60^{\circ} \mathrm{C}$ and a flow rate of $0.6 \mathrm{~mL} \mathrm{~min}^{-1}$ of $5 \mathrm{mM} \mathrm{H}_{2} \mathrm{SO}_{4}$.

The total lipid extraction method was adapted from Bligh and Dyer (Bligh and Dyer 1959). Briefly, $15 \mathrm{mg}$ of freezedried cell pellets were treated with 1 unit $\mu \mathrm{L}^{-1}$ zymolyase in $1 \mathrm{~mL}$ digestion buffer (1.2 M glycerol, $100 \mathrm{mM}$ sodium thioglycolate, $50 \mathrm{mM}$ Tris-sulfate, $\mathrm{pH} 7.5$ ) at $37^{\circ} \mathrm{C}$ for $15 \mathrm{~min}$, followed by centrifugation at $3000 \mathrm{rpm}$ for $3 \mathrm{~min}$. The mixture of collected spheroplasts was spiked with cholesterol as internal standard, $7 \mathrm{ml}$ of chloroform:methanol $(2: 1, \mathrm{v} / \mathrm{v})$ were added and shaken vigorously at room temperature and $500 \mathrm{rpm}$ for $30 \mathrm{~min} .1 .7 \mathrm{~mL}$ of $0.73 \%$ 
$\mathrm{NaCl}$ was added to the mixture prior centrifugation at $3500 \mathrm{rpm}$ for $5 \mathrm{~min}$ at $4^{\circ} \mathrm{C}$. The lower (organic) phase was collected and the upper phase was re-extracted with $5 \mathrm{~mL}$ of chloroform-methanol $(85: 15 \mathrm{v} / \mathrm{v})$. The lower (organic) phase was collected and pooled with the previous organic fraction and kept at $-20^{\circ} \mathrm{C}$ for further HPLC analysis.

Lipid separation and quantification were performed using the method modified from Silversand and Haux (Silversand and Haux 1997). Lipid separation was accomplished using an Ultimate 3000 HPLC (Dionex) equipped with a Corona charged aerosol detector (CAD) (Dionex) connected with nitrogen gas at 35 psi gas pressure. A $20 \mu \mathrm{L}$ volume of each sample was injected into a Luna $5 \mu \mathrm{m}$ HILIC $200 \AA 250 \times 4.6 \mathrm{~mm} \mathrm{LC}$ column (Phenomenex, Torrance, CA). The flow-rate was $0.8 \mathrm{~mL} \cdot \mathrm{min}^{-1}$ and the column temperature was kept at $25^{\circ} \mathrm{C}$ during all runs. A gradient flow with 3 solvents (solvent A, 99:1 by vol. of hexane/acetic acid, solvent B 70:29:1, by vol. of hexane/isopropanol/acetic acid, solvent C 85:14:1, by vol. of isopropanol/water/acetic acid) was applied for the separation of lipids. Triethylamine (0.08\%) was added to solvent B and solvent C for better separation. The HPLC system was equilibrated with $99 \%$ solvent A. The gradient profile started from 3\% solvent $\mathrm{B}$ at $7 \mathrm{~min}$, reached $10 \%$ solvent $\mathrm{B}$ in $1 \mathrm{~min}$ and ended at $100 \%$ solvent $B$ in 15 min, while solvent $C$ was kept at $0 \%$ from $0 \mathrm{~min}$ to $15 \mathrm{~min}$. This gradient profile is used for separation of triacylglycerol (TAG), free fatty acids, cholesterol (as internal standard) and ergosterol. After 15 min, solvent A was kept at $0 \%$ until 35 min. Meanwhile, an increase of solvent $\mathrm{C}$ to $65 \%$ from $15 \mathrm{~min}$ to 32 min was applied for separation of phospholipids (phosphatidylethanolamine, phosphatidylinositol, phosphatidylcholine, phosphatidylserine, phosphatidic acid). After that, solvent $\mathrm{C}$ was decreased to $0 \%$ from $32 \mathrm{~min}$ to $35 \mathrm{~min}$ and $100 \%$ solvent B was applied to the system. After $40 \mathrm{~min}$, 99\% solvent A was run for $5 \mathrm{~min}$ in order to equilibrate and stabilize the system for the measurement of the next sample. A logarithmic plot of peak area versus the concentration of each lipid standard was used to generate a calibration curve. The slope determined from the log-log plot was further used for lipid quantification (Nair and Werling 2009).

\section{Results}

\section{Characterization of PHB-producing S. cerevisiae}

The engineered $S$. cerevisiae strains were preliminary studied for growth and PHB production in shake flasks as shown in Figure 2. Co-expression of beta-ketothiolase, acetoaetyl-CoA reductase and PHA synthase results in PHB accumulation in S. cerevisiae as observed in previous studies (Breuer et al. 2002; Carlson et al. 2002; Carlson and Srienc 2006). In this study, the 3 genes involved in the PHB pathway were expressed from a single vector in order to avoid the heterogeneity of plasmid distribution. S. cerevisiae carrying the PHB plasmid and an empty HIS3 plasmid (strain SCKK005) and a strain carrying the $\mathrm{PHB}$ plasmid and the acetyl-CoA boost plasmid (SCKK006) were characterized and evaluated for the productivity of PHB. The acetyl-CoA boost plasmid contains 4 genes, $A D H 2, A L D 6$, acs ${ }^{\mathrm{L} 641 \mathrm{P}}$ and $E R G 10$, involved in channeling carbon from ethanol to acetyl-CoA. In the acetyl-CoA boost plasmid, ALD6, acs $^{\text {L641P }}$ and ERG10 are controlled by constitutive promoters, $\mathrm{P}_{T E F 1}$ and $\mathrm{P}_{P G K 1}$, respectively, while $A D H 2$ is under control of the $\mathrm{P}_{H X T 7}$ promoter which is strongly de-repressed under glucose depletion (Partow et al. 2010; Reifenberger et al. 1995; Sedlak and Ho 2004).

CIT2 encoding peroxisomal citrate synthase catalyzes the conversion of oxaloacetate and acetyl-CoA to citrate and plays role in acetate metabolism. MLS1 encoding malate synthase catalyzes the conversion of glyoxylate and acetylCoA to malate in the glyoxylate shunt. Deletion of CIT2 and MLS1 affect the integrity of the glyoxylate shunt and will therefore reduce the drain of acetyl-CoA through this pathway and hereby possibly affect the availability of cytosolic acetyl-CoA. Thus, the effect of CIT2 and MLS1 deletion on PHB production was also investigated. The biomass yield of cit2 $\Delta$ (SCKK009) was less than that of $m l s 1 \Delta$ (SCKK010), and the biomass yield for both the deletion strains were lower than those of the non-deletion reference strain without the acetyl-CoA plasmid (SCKK005) and the non-deletion strain with the acetyl-CoA plasmid (SCKK006) (see Figure 2a). The biomass yield of the deletion strains, SCKK009 and SCKK010, was lower than that of non-deletion strains, SCKK005 and SCKK006, due to the impaired $\mathrm{C}_{2}$ carbon utilization. The recombinant strain with both the acetyl-CoA boost plasmid and the PHB plasmid (SCKK006) produced an 18 times higher final concentration of PHB (at $120 \mathrm{~h}$ ) compared to the reference strain, SCKK005 (Figure 2b). Besides that, the amount of accumulated PHB in SCKK009 and SCKK010 was less than SCKK006. Although, mls1 1 (SCKK010) gave lower biomass yield than the reference, it gave higher $\mathrm{PHB}$ titer compare to the non-deletion reference strain (SCKK005) when the deletion strain carried the acetyl-CoA plasmid. This result showed the combined effect of $m l s 1 \Delta$ together with the utilization of acetyl-CoA plasmid on the availability of acetyl-CoA and the influence of the deletion on PHB production. The consumption rate of ethanol in the deletion strains, especially in cit2 $\triangle$ (SCKK009), was slower than for the non-deletion strain as the residual amount of ethanol was detected after $80 \mathrm{~h}$ of cultivation (Figure 2c). In addition, more than $6.5 \mathrm{~g} \mathrm{~L}^{-1}$ of acetate was detected in the medium after 120 of fermentation of SCKK009, which is clear evidence for the impaired $\mathrm{C}_{2}$ metabolism. Due to the negative impact of CIT2 and MLS1 deletion on growth and PHB production, the recombinant strains SCKK005 and 
Figure 2 Measurements of biomass and PHB from shake flask cultivations in a modified minimal medium with $20 \mathrm{~g} \mathrm{~L}^{-1}$ glucose as carbon source. Strain SCKK005 harbors an empty plasmid (plYC04) and the PHB plasmid (pKK01), strain SCKKO06 harbors an acetyl-COA boost plasmid (pIYC08) and the PHB plasmid (pKK01), SCKK009 and SCKK010 harbor plYC08 and pKK01 and carry a CIT2 and MLS1 deletion, respectively.

SCKK006 without gene deletions were selected for further characterization in bioreactors.

Kinetic studies of the PHB-producing S. cerevisiae strains were carried out in aerobic bioreactor cultivation. The production and accumulation of PHB coincided with the depletion of glucose and the increase in ethanol concentration during the glucose consumption phase. SCKK005 and SCKK006 demonstrated a similar growth profile as shown in Figure 3. Kinetic parameters and yields on glucose and ethanol are summarized in Table 3. There was no significant difference in the maximum specific growth rates of SCKK005 and SCKK006, which were $0.27 \pm 0.02 \mathrm{~h}^{-1}$ and $0.28 \pm 0.00 \mathrm{~h}^{-1}$, respectively. The glucose consumption rate of SCKK006 was higher than that of SCKK005. However, a slightly lower biomass yield on glucose of SCKK006 was observed. The strain carrying the acetyl-CoA boost plasmid showed the capability to increase the carbon flux from ethanol to the PHB pathway as the PHB yield on ethanol in SCKK006 was significantly higher than that of SCKK005 In the ethanol phase, the PHB yield on ethanol in SCKK006 was 6.09 $\pm 1.44 \mathrm{mg}(\mathrm{g} \mathrm{EtOH})^{-1}$, which was approximately 25-fold higher than for SCKK005 that had a yield of $0.22 \pm 0.04 \mathrm{mg}(\mathrm{g} \mathrm{EtOH})^{-1}$. The maximum $\mathrm{PHB}$ titer detected during the ethanol phase in SCKK005 was $1.85 \mathrm{mg} \cdot \mathrm{L}^{-1}$ while SCKK006, which contained both the PHB biosynthesis and the acetyl-CoA boost plasmid, reached a titer of $43.11 \mathrm{mg} \cdot \mathrm{L}^{-1}$ after $36 \mathrm{~h}$ of batch fermentation. In SCKK005, the PHB level remained at the same concentration until the end of fermentation while the PHB titer in SCKK006 tended to decrease after $50 \mathrm{~h}$.

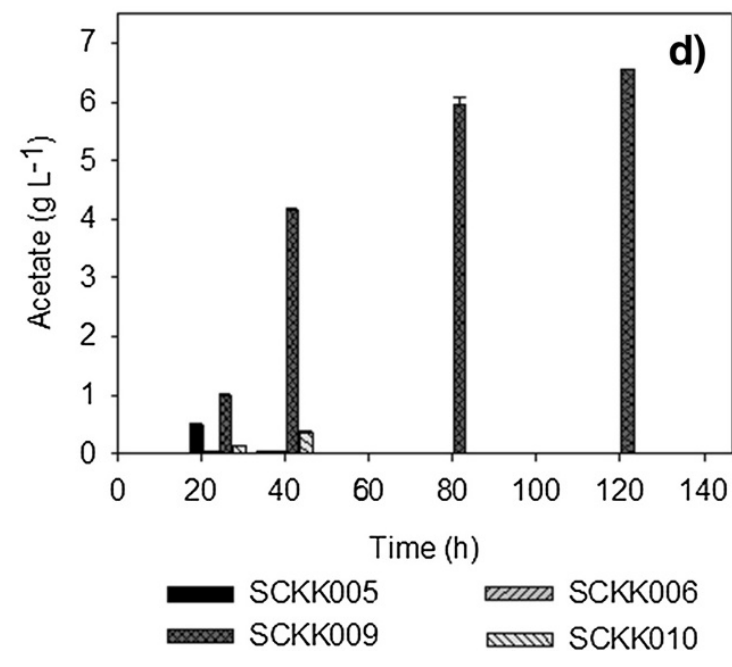

\section{Comparison of specific productivities in pathway engineered strains}

The effect of the acetyl-CoA boost plasmid was clearly seen during growth in the glucose phase when SCKK006 started to produce and accumulate PHB with a yield of PHB on glucose of $0.13 \pm 0.02 \mathrm{mg}\left(\mathrm{g} \mathrm{glc}^{-1}{ }^{-1}\right.$ higher than that of SCKK005 as previously shown in Table 3. Figure 4 shows the consumption of glucose and the formation of ethanol, glycerol and acetate measured during growth in an aerobic batch bioreactor. A maximum glycerol concentration of $1.33 \pm 0.06 \mathrm{~g} \cdot \mathrm{L}^{-1}$ was observed in SCKK006, while a lower glycerol concentration, $0.86 \pm 0.01 \mathrm{~g} \cdot \mathrm{L}^{-1}$, was observed in SCKK005. This result indicates the influence of $A D H 2$ overexpression in order to convert ethanol to acetaldehyde thus resulting in a small increase in $\mathrm{NADH}$ 


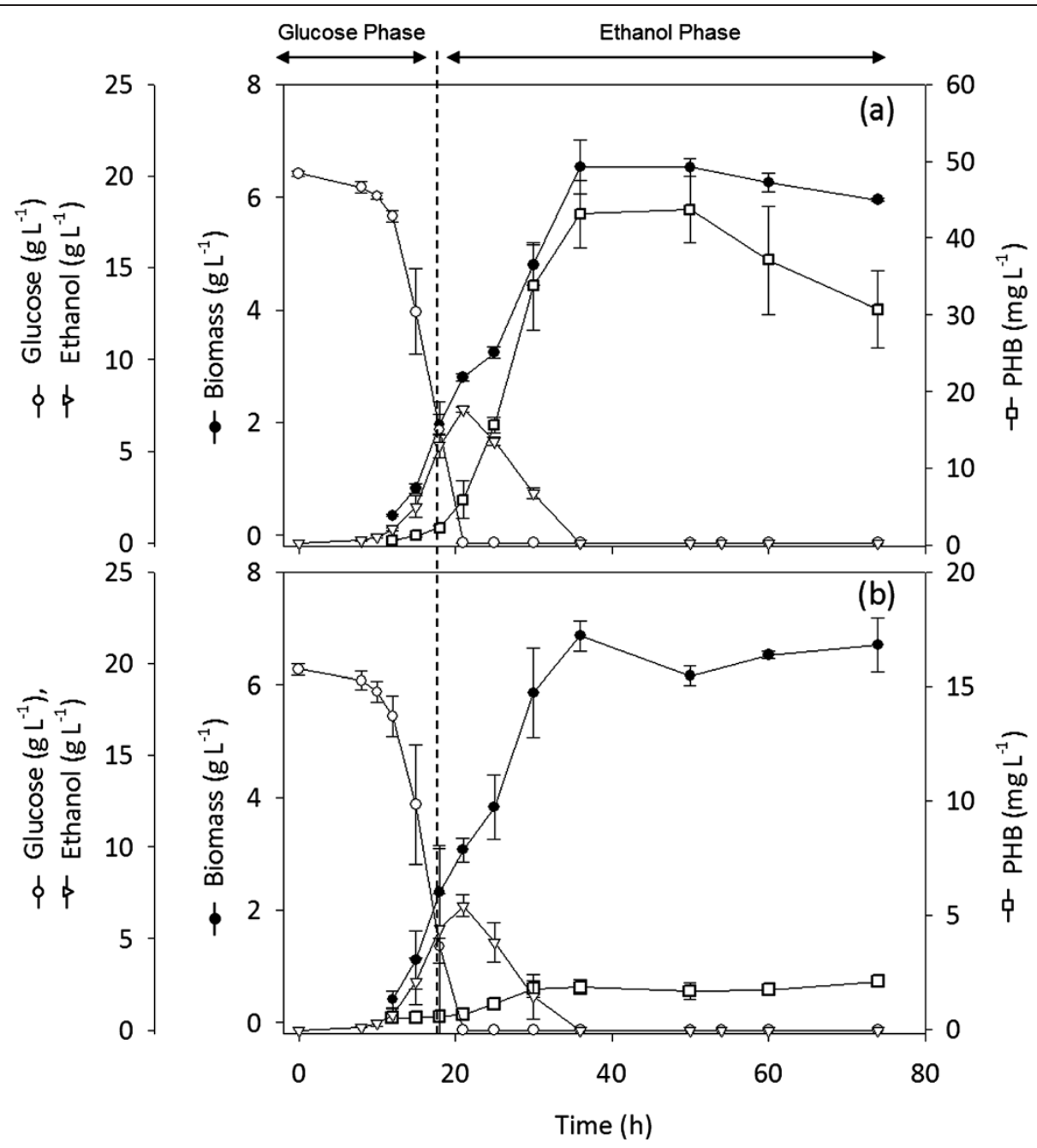

Figure 3 Fermentation profile of $S$. cerevisiae producing PHB in aerobic batch bioreactor cultivation using a chemically defined medium with $20 \mathrm{~g} \mathrm{~L}^{-1}$ glucose as carbon source. (a) SCKK006: S. cerevisiae harboring an acetyl-CoA boost plasmid (plYC08) and the PHB plasmid (pKK01). (b) SCKK005: S. cerevisiae harboring an empty plasmid (plYC04) and the PHB plasmid (pKK01).

production, which triggers the formation of glycerol for redox balancing in the cytosol (Bakker et al. 2001). On the other hand, the maximum acetate concentration of $0.42 \pm 0 \mathrm{~g} \cdot \mathrm{L}^{-1}$ detected in SCKK005 was 3 times higher than the acetate concentration of $0.12 \pm 0.01 \mathrm{~g} \cdot \mathrm{L}^{-1}$ detected in SCKK006. Therefore, the different concentrations of glycerol and acetate detected during growth on glucose are most likely a consequence of introducing the acetyl-CoA boost plasmid in the PHB producing strain.

To further explore carbon channeling from glucose to PHB and use of acetyl-CoA for lipid synthesis we measured phospholipids, triacylglycerols, free fatty acids and ergosterol in the cells. Using these measurements we calculated the specific productivities $\left(r_{p}\right)$ of ethanol, glycerol, PHB, triacylglycerols (TAG), ergosterol, phospholipids and free fatty acids as directly and non-directly acetyl-CoA derived products during growth on glucose using the maximum specific growth rate $\left(\mu_{\max }\right)$ and the product yield coefficient.
Table 3 Yields and kinetic parameters obtained from batch cultivations

\begin{tabular}{|c|c|c|c|}
\hline & Unit & SCKK005 & SCKK006 \\
\hline Maximum specific growth rate & $h^{-1}$ & $0.27 \pm 0.02$ & $0.28 \pm 0.00$ \\
\hline Glucose consumption rate & $g(g \text { DW h })^{-1}$ & $1.80 \pm 0.09$ & $2.24 \pm 0.33$ \\
\hline Biomass yield on glucose & $g(g \mathrm{glc})^{-1}$ & $0.15 \pm 0.01$ & $0.13 \pm 0.02$ \\
\hline Ethanol yield on glucose & $g(g g \mid c)^{-1}$ & $0.35 \pm 0.05$ & $0.35 \pm 0.07$ \\
\hline Glycerol yield on glucose ${ }^{*}$ & $g(g \mathrm{glc})^{-1}$ & $0.05 \pm 0.00$ & $0.07 \pm 0.00$ \\
\hline Acetate yield on glucose ${ }^{*}$ & $g(g \mathrm{glc})^{-1}$ & $0.02 \pm 0.00$ & 0 \\
\hline PHB yield on glucose ${ }^{*}$ & $\mathrm{mg}(\mathrm{g} \mathrm{glc})^{-1}$ & $0.02 \pm 0.01$ & $0.13 \pm 0.02$ \\
\hline Biomass yield on ethanol & $g(g \mathrm{EtOH})^{-1}$ & $0.46 \pm 0.27$ & $0.45 \pm 0.08$ \\
\hline PHB yield on ethanol ${ }^{*}$ & $m g(g \mathrm{EtOH})^{-1}$ & $0.22 \pm 0.04$ & $6.09 \pm 1.44$ \\
\hline
\end{tabular}

The values were calculated from at least triplicate fermentations $(n \geq 3)$ and represent as mean \pm SD.

* The values are significantly difference at $p$-value $\leq 0.05$. 


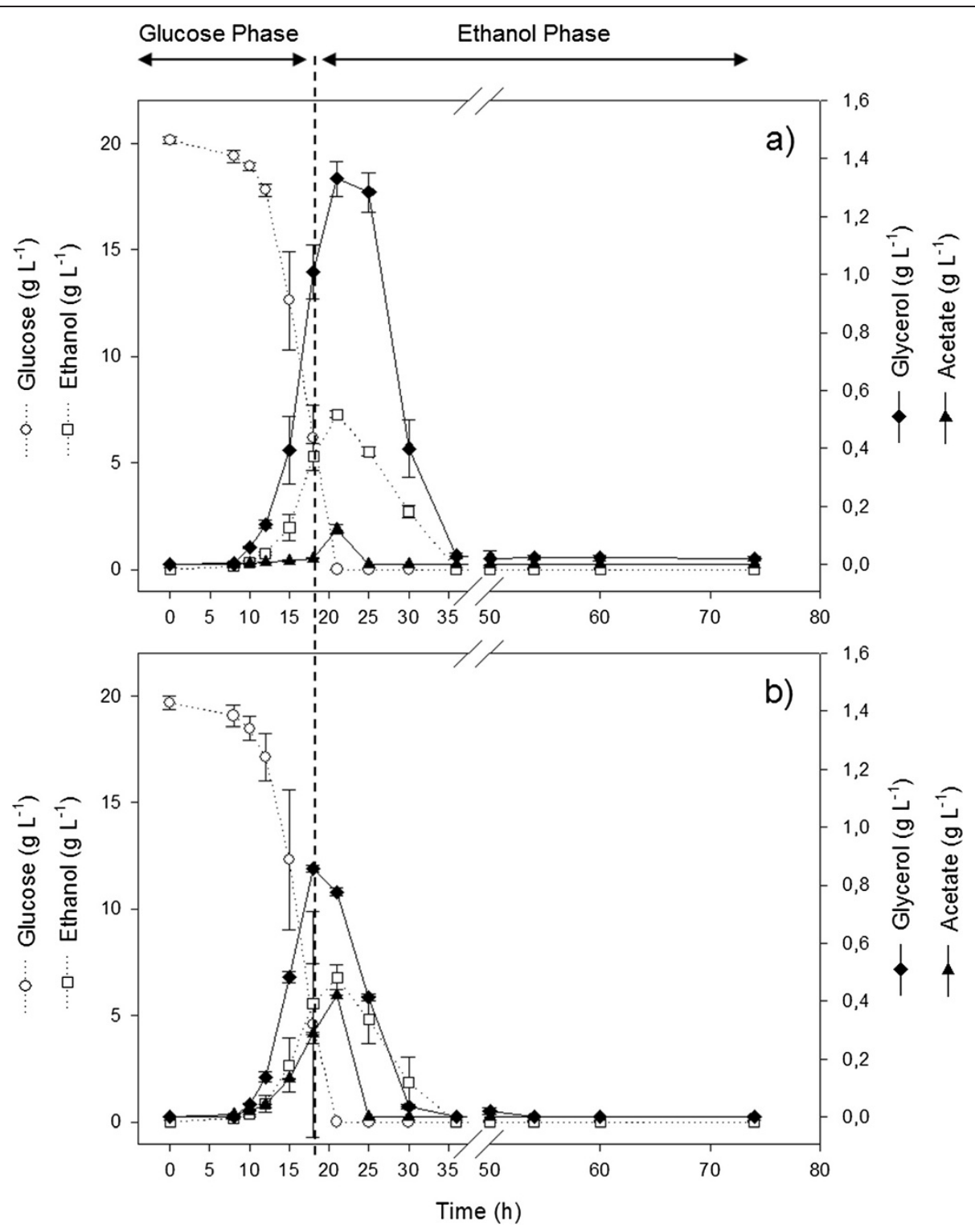

Figure 4 Time profile of glucose consumption and ethanol, glycerol and acetate formation during batch bioreactor cultivation of a) SCKK006 and b) SCKK005 in a chemically define minimal medium with $20 \mathrm{~g} \mathrm{~L}^{-1}$ glucose as carbon source. SCKK006: S. cerevisiae harboring an acetyl-CoA boost plasmid (plYC08) and the PHB plasmid (pKK01). and SCKK005: S. cerevisiae harboring an empty plasmid (plYC04) and the PHB plasmid (pKK01), respectively.

The biomass yield coefficient $\left(\mathrm{Y}_{\mathrm{sx}}\right)$ and the product yield coefficient $\left(\mathrm{Y}_{\mathrm{sp}}\right)$ are defined as the amount of substrate $(\mathrm{s})$ consumed for the formation of biomass ( $\mathrm{x}$ ) or other metabolites (p) such as ethanol, acetate or PHB. The specific product formation rate was calculated by using the equation: $r_{p}=$ $\mu_{\max } \cdot Y_{\mathrm{sp}}$ and the specific glucose consumption rate was calculated by using the equation: $r_{s}=\frac{\mu_{\max }}{Y_{\mathrm{sx}}}$. Figure 5 illustrates a simplified pathway with the calculated fluxes during growth on glucose. The mean value \pm SD from at least triplicate fermentations is reported. The carbon from glucose was mainly directed to ethanol as the major product in $S$. cerevisiae. The impact of using the acetyl-CoA boost plasmid was clearly associated with a higher specific productivity of PHB in SCKKO06 as well as the lower specific productivities of triacylglycerol and phospholipids as other acetylCoA derived products. The higher specific productivity of glycerol in SCKK006 revealed the collateral effect of $A D H 2$ over-expression as mentioned above. The specific productivity of ergosterol, which is also derived from acetoacetyl$\mathrm{CoA}$, an intermediate in the PHB pathway, was lower in SCKK006 than in SCKK005, which shows that the heterologous PHB pathway is clearly able to compete for acetoacetyl-CoA otherwise used for biosynthesis of 


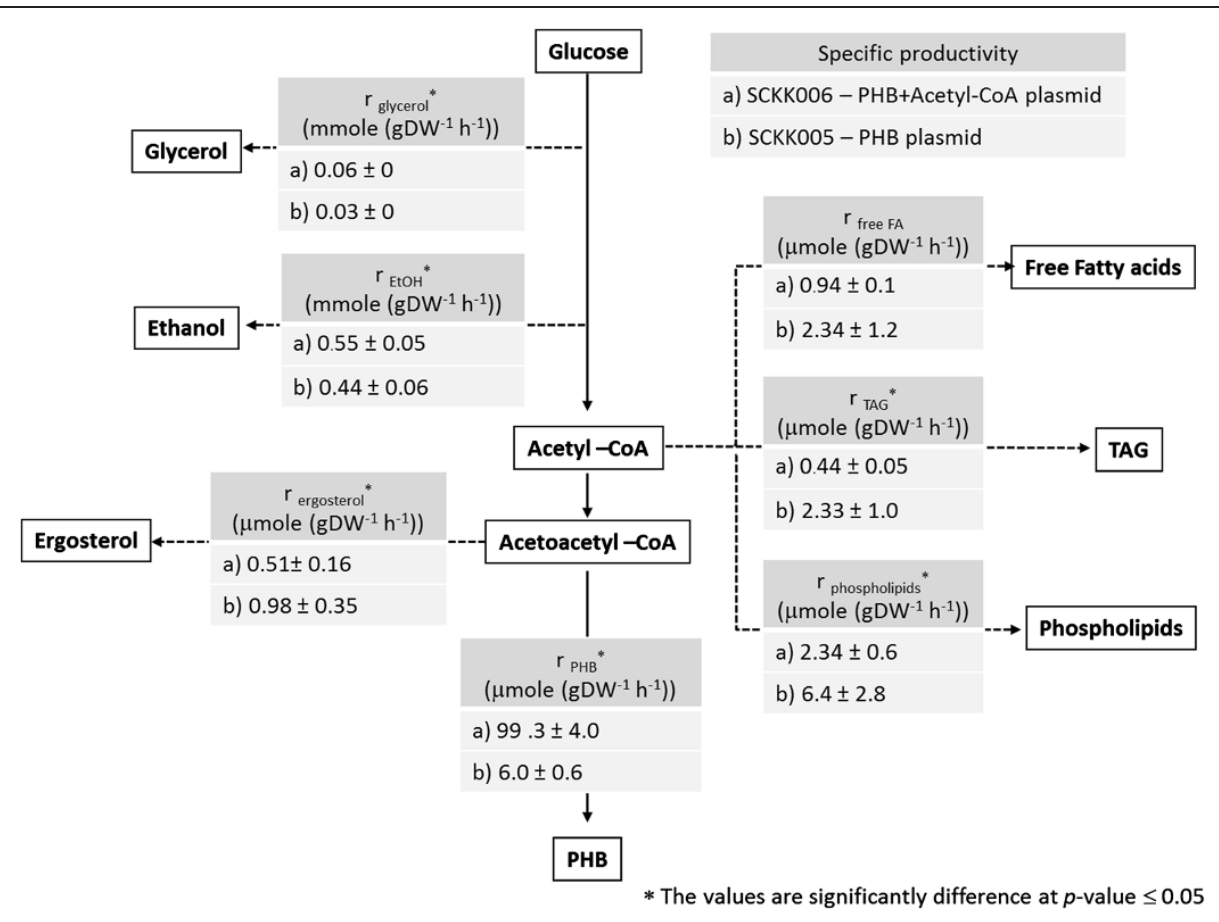

Figure 5 Comparison of specific fluxes in SCKK006 and SCKK005 during growth on glucose in aerobic batch bioreactor cultivation with $20 \mathrm{~g} \mathrm{~L}^{-1}$ glucose as carbon source. SCKK006 is S. cerevisiae harboring an acetyl-CoA boost plasmid (plYC08) and the PHB plasmid (pKK01). SCKKO05 is S. cerevisiae harboring an empty plasmid (plYCO4) and the PHB plasmid (pKK01). The fluxes towards the different lipids were calculated from measurement of the lipid composition of the biomass and the maximum specific growth rate. The mean value \pm SD from at least triplicate fermentations are reported.

ergosterol. Finally, as a result of employing the acetyl-CoA boost plasmid to channel carbon from ethanol to the $\mathrm{PHB}$ pathway, the specific productivity of $\mathrm{PHB}$ in the glucose phase in the strain carrying the acetyl-CoA boost plasmid (SCKK006) was $99.3 \pm 4 \mu$ mole $\left(\mathrm{g} \mathrm{DW}^{-1} \mathrm{~h}^{-1}\right), 16.5$ times higher compared to the strain carrying the empty acetylCoA plasmid (SCKK005).

\section{Discussion}

The addition of precursor molecules to the fermentation medium has been shown to improve the formation of many biological products, e.g. supplementation with glucosamine as precursor resulted in improved hyaluronic acid production by $E$. coli (Mao et al. 2009) and supplementation with specific amino acids enhanced heterologous protein production by S. cerevisiae (Görgens et al. 2005). Extra feeding of acetate $\left(0.5 \mathrm{~g} \cdot \mathrm{L}^{-1}\right.$ with $\mathrm{pH}$ adjusted to 4.5$)$ and panthothenate $\left(1000 \mu \mathrm{g} \cdot \mathrm{L}^{-1}\right)$ as a precursors of acetyl-CoA were tested by Carlson and Srienc in order to increase PHB accumulation in S. cerevisiae (Carlson and Srienc 2006). Feeding a combination of acetate and pantothenate was found to improve $\mathrm{PHB}$ production by approximately $45 \%$ over the control. Instead of feeding precursors to improve the productivity, which would increase the costs of PHB production, we focused on pathway engineering to increase the supply of the precursor for $\mathrm{PHB}$ production and hereby improve the economic feasibility of a process using $S$. cerevisiae.

The native $A D H 2$ in $S$. cerevisiae is a glucose-repressible alcohol dehydrogenase. It catalyzes the oxidation of ethanol to acetaldehyde only when glucose becomes depleted from the medium. In order to activate the reaction catalyzed by $A D H 2$ when glucose was completely consumed, the $H X T 7$ promoter was selected to control $A D H 2$ in the acetyl-CoA plasmid. Although the $H X T 7$ promoter is a glucose-repressible promoter, we select the HXT7 promoter based on its strong expression level especially during the ethanol phase. Therefore, $A D H 2$ under control of the HXT7 promoter ensures the conversion of ethanol to acetyldehyde and is responsible for the initial step in the utilization of ethanol as carbon source. ALD6 encoding cytosolic aldehyde dehydrogenase is involved in the conversion of acetaldehyde to acetate. This enzyme utilizes $\mathrm{NADP}^{+}$as the preferred coenzyme. As a result, ALD6 over-expression might help providing $\mathrm{NADPH}$, a required cofactor for PHB production (Boubekeur et al. 2001). There are two acetyl-CoA synthetase in $S$. cerevisiae, encoded by ACS1 and ACS2. Both of these enzymes catalyze ATP-dependent activation of acetate to acetylCoA. The expression of ACS1 is subjected to glucose repression while $A C S 2$ can be expressed during growth on glucose where it is likely to be responsible for cytosolic 
acetyl-CoA production. However, the regulation of both enzymes is complex and hence over-expression is believed necessary to increase the flux towards acetyl-CoA. In the native host Salmonella enterica, mutations at Leu-641 of acetyl CoA synthetase (Acs), prevent the acetylation by acetyltransferase bypassing the need for sirtuin deacetylase activity and maintain the enzyme in an active state during growth on acetate. (Starai et al. 2005). Enhancing acetylCoA supply by engineering the pyruvate dehydrogenase bypass through over-expression of acetaldehyde dehydrogenase in combination with introduction of a $S$. enterica acetyl CoA synthetase variant (L641P) in S. cerevisiae, was successful demonstrated to improve the productivity of isoprenoids in yeast (Shiba et al. 2007).

CIT2, the peroxisomal citrate synthase and MLS1, the cytosolic malate synthase are key enzymes of the glyoxylate shunt and hereby affect acetate metabolism (Kunze et al. 2006; Lee et al. 2011). Deletion of CIT2 and MLS1 in order to improve the availability of cytosolic acetyl-CoA was investigated for its impact on PHB production. However, this deletion strategy resulted in the impaired metabolism due to the incapability to utilize $\mathrm{C}_{2}$ carbon via the glyoxylate shunt in cit $2 \Delta$ and $m l s 1 \Delta$ strains. The deletion strains have only the tricarboxylic acid cycle (TCA cycle) to utilize $\mathrm{C}_{2}$ carbon for energy production whereas the biosynthesis of $\mathrm{C}_{4}$ dicarboxylic acids required as precursors for amino acids biosynthesis cannot take place, resulting in lack of growth (Chen et al. 2012). This is reflected by a much reduced biomass yield in the cit $2 \Delta$ strain. In the $m l s 1 \Delta$ strain (SCKK009), less acetate was accumulated in the medium compared to the cit $2 \Delta$ strain and acetate was slowly consumed until the end of fermentation. This might be due to the activity of the homolog malate synthase encoded by DAL7 (Hartig et al. 1992). Moreover, the deletion of CIT2 affect acetate metabolism and reduced the efficiency of acetyl-CoA synthetase, both native acetyl-CoA synthetase and the additional acetyl-CoA synthetase, to catalyze the ATP-dependent activation of acetate to acetylCoA thus resulting in more than $6.5 \mathrm{~g} / \mathrm{L}^{-1}$ of acetate accumulated in the cit $2 \Delta$ strain and no further biomass production after glucose is completely consumed (after $24 \mathrm{hr}$ ). Besides that, the PHB titer was drastically decreased as compared to the non-deletion strain, SCKK006.

The comparison of the specific productivities in the $\mathrm{PHB}$ producing strains revealed that employing the acetyl-CoA boost plasmid helps in directing carbon towards the $\mathrm{PHB}$ pathway. Although, there was a difference in $\mathrm{PHB}$ yield when the PHB strains, SCKK005 and SCKK006, were cultivated in bioreactors. This might be explained by the cultivation condition in the bioreactor which favors the growth of $S$. cerevisiae rather than PHB production. Thus, the maximum specific growth rate in shake flasks was $0.18-0.20 \mathrm{~h}^{-1}$ while the maximum specific growth rate in the bioreactors was 0.27-0.28 $\mathrm{h}^{-1}$. Although the PHB yield from the bioreactors was less than shake flask cultivation the difference in PHB productivity between SCKK005 and SCKK006 clearly showed the effect of employing the acetyl-CoA plasmid for PHB production. Therefore, the flux from glucose to PHB was analyzed by using the information from aerobic batch bioreactor cultivations. For this analysis free fatty acids, TAG, phospholipids and ergosterol were considered as side products of PHB production. From this analysis we found that over-expression of ERG10 together with phaA significantly reduced the specific productivities of TAG and phospholipids as side products compared to the reference strain which does not have over-expression of ERG10. Although increased production of acetyl-CoA might lead to fatty acid or lipid synthesis, our study show that when the cells contain a heterologous pathway to PHB flux is actually directed towards $\mathrm{PHB}$ rather than towards lipids. Thus, we can conclude that enhancement of acetyl-CoA production by co-expression of genes on the acetyl-CoA boost plasmid improved the productivity of $\mathrm{PHB}$ during growth on glucose and further enhanced the productivity of PHB approximately 16.5 times bioreactor cultivations and reduce the flux from acetyl-CoA to lipids.

\section{Competing interests}

The authors declare that they have no competing interest.

\section{Authors' contributions}

JN and KK participated in the design of the study. JN and VS supervised the project and edited manuscript. YC contributed the plasmid, plYC08, used in this study. KK performed the experimental work, analyzed the data and wrote the manuscript. All authors read and approved the final manuscript.

\section{Acknowledgements}

We thank Chalmers Foundation and the Knut and Alice Wallenberg Foundation for funding part of this work. We also acknowledge the Thailand Science and Technology Ministry for providing a stipend to KK.

Received: 8 September 2012 Accepted: 12 September 2012 Published: 25 September 2012

\section{References}

Bakker BM, Overkamp KM, van Maris AJA, Kötter P, Luttik MAH, van Dijken JP, Pronk JT (2001) Stoichiometry and compartmentation of NADH metabolism in Saccharomyces cerevisiae. FEMS Microbiol Rev 25(1):15-37

Bertani G (1951) Studies on lysogenesis I. J Bacteriol 62(3):293-300

Bligh EG, Dyer WJ (1959) A rapid method of total lipid extraction and purification. Can J Biochem Physiol 37(8):911-917

Bohmert K, Balbo I, Kopka J, Mittendorf V, Nawrath C, Poirier Y, Tischendorf G, Trethewey RN, Willmitzer L (2000) Transgenic Arabidopsis plants can accumulate polyhydroxybutyrate to up to $4 \%$ of their fresh weight. Planta 211(6):841-845

Boubekeur S, Camougrand N, Bunoust O, Rigoulet M, Guérin B (2001) Participation of acetaldehyde dehydrogenases in ethanol and pyruvate metabolism of the yeast Saccharomyces cerevisiae. Eur J Biochem 268(19):5057-5065

Breuer U, Terentiev Y, Kunze G, Babel W (2002) Yeasts as producers of polyhydroxyalkanoates: Genetic engineering of Saccharomyces cerevisiae. Macromol Bioscie 2(8):380-386

Carlson R, Fell D, Srienc F (2002) Metabolic pathway analysis of a recombinant yeast for rational strain development. Biotechnol Bioeng 79(2):121-134

Carlson R, Srienc F (2006) Effects of recombinant precursor pathway variations on poly[(R)-3-hydroxybutyrate] synthesis in. J Biotechnol 124(3):561-573

Chen Y, Siewers V, Nielsen J (2012) Profiling of cytosolic and peroxisomal acetylCoA metabolism in Saccharomyces cerevisiae. PLoS One 7(8):e42475 
de Almeida A, Giordano AM, Nikel PI, Pettinari MJ (2010) Effects of aeration on the synthesis of poly(3-hydroxybutyrate) from glycerol and glucose in recombinant Escherichia coli. Appl Environ Microbiol 76(6):2036-2040

Dimster-Denk D, Rine J (1996) Transcriptional regulation of a sterol-biosynthetic enzyme by sterol levels in Saccharomyces cerevisiae. Mol Cell Biol 16(8):3981-3989

Gietz RD, Woods RA (2002) Transformation of yeast by lithium acetate/single-stranded carrier DNA/polyethylene glycol method. Methods Enzymol 350:87-96

Goffeau A (2000) Four years of post-genomic life with 6000 yeast genes. FEBS Lett 480(1):37-41

Görgens JF, van Zyl WH, Knoetze JH, Hahn-Hägerdal B (2005) Amino acid supplementation improves heterologous protein production by Saccharomyces cerevisiae in defined medium. Appl Microbiol Biotechnol 67(5):684-691

Hankermeyer CR, Tjeerdema RS (1999) Polyhydroxybutyrate: Plastic made and degraded by microorganisms. In: Ware GW (ed) Revi Environ Contam Toxicol, vol 159. Springer, New York, pp 1-24

Hartig A, Simon MM, Schuster T, Daugherty JR, Yoo HS, Cooper TG (1992) Differentially regulated malate synthase genes participate in carbon and nitrogen metabolism of S. cerevisiae. Nucleic Acids Res 20(21):5677-5686

Hiser L, Basson ME, Rine J (1994) ERG10 from Saccharomyces cerevisiae encodes acetoacetyl-CoA thiolase. J Biol Chem 269(50):31383-31389

Karr DB, Waters JK, Emerich DW (1983) Analysis of poly-betahydroxybutyrate in Rhizobium japonicum bacteroids by ion-exclusion high-pressure liquid chromatography and UV detection. Appl Environ Microbiol 46(6):1339-1344

Kunze M, Pracharoenwattana I, Smith SM, Hartig A (2006) A central role for the peroxisomal membrane in glyoxylate cycle function. Biochim Biophys Acta 1763(12):1441-1452

Leaf TA, Peterson MS, Stoup SK, Somers D, Srienc F (1996) Saccharomyces cerevisiae expressing bacterial polyhydroxybutyrate synthase produces poly3-hydroxybutyrate. Microbiol 142(Pt 5):1169-1180

Lee YJ, Jang JW, Kim KJ, Maeng PJ (2011) TCA cycle-independent acetate metabolism via the glyoxylate cycle in Saccharomyces cerevisiae. Yeast 28(2):153-166

Mao Z, Shin H-D, Chen R (2009) A recombinant E. coli bioprocess for hyaluronan synthesis. Appl Microbiol Biotechnol 84(1):63-69

Marchesini S, Erard N, Glumoff T, Hiltunen JK, Poirier Y (2003) Modification of the monomer composition of polyhydroxyalkanoate synthesized in Saccharomyces cerevisiae expressing variants of the beta-oxidation-associated multifunctional enzyme. Appl Environ Microbiol 69(11):6495-6499

Melchiors M, Keul H, Höcker H (1994) Synthesis of highly isotactic poly[(R)-3hydroxybutyrate] by ring-opening polymerization of $(R, R, R)-4,8,12-$ trimethyl-1,5,9-trioxacyclododeca-2,6,10-trione. Macromol Rapid Commun 15(6):497-506

Nair LM, Werling JO (2009) Aerosol based detectors for the investigation of phospholipid hydrolysis in a pharmaceutical suspension formulation. J Pharm Biomed Anal 49(1):95-99

Nawrath C, Poirier Y, Somerville C (1994) Targeting of the polyhydroxybutyrate biosynthetic pathway to the plastids of Arabidopsis thaliana results in high levels of polymer accumulation. PNAS 91(26):12760-12764

Nikel PI, Pettinari MJ, Galvagno MA, Méndez BS (2006) Poly(3-hydroxybutyrate) synthesis by recombinant Escherichia coli arcA mutants in microaerobiosis. Appl Environ Microbioly 72(4):2614-2620

Nookaew I, Jewett MC, Meechai A, Thammarongtham C, Laoteng K, Cheevadhanarak S, Nielsen J, Bhumiratana S (2008) The genome-scale metabolic model ilN800 of Saccharomyces cerevisiae and its validation: a scaffold to query lipid metabolism. BMC Syst Biol 2:71

Partow S, Siewers V, Bjorn S, Nielsen J, Maury J (2010) Characterization of different promoters for designing a new expression vector in Saccharomyces cerevisiae. Yeast 27(11)):646-955

Peoples OP, Sinskey AJ (1989a) Poly-beta-hydroxybutyrate (PHB) biosynthesis in Alcaligenes eutrophus H16. Identification and characterization of the PHB polymerase gene (phbC). J Biol Chem 264(26):15298-15303

Peoples OP, Sinskey AJ (1989b) Poly-beta-hydroxybutyrate biosynthesis in Alcaligenes eutrophus $\mathrm{H} 16$, Characterization of the genes encoding betaketothiolase and acetoacetyl-CoA reductase. J Biol Chem 264(26):15293-15297

Petrasovits LA, Zhao L, McQualter RB, Snell KD, Somleva MN, Patterson NA, Nielsen LK, Brumbley SM (2012) Enhanced polyhydroxybutyrate production in transgenic sugarcane. Plant Biotechnol J 10(5):569-578

Poirier Y, Dennis D, Klomparens K, Nawrath C, Somerville C (1992) Perspectives on the production of polyhydroxyalkanoates in plants. FEMS Microbiol Lett 103(2-4):237-246
Reifenberger E, Freidel K, Ciriacy M (1995) Identification of novel HXT genes in Saccharomyces cerevisiae reveals the impact of individual hexose transporters on glycolytic flux. Mol Microbiol 16(1):157-167

Sambrook J, Russell DW (2006) The Inoue method for preparation and transformation of competent E. Coli: "Ultra-competent" cells. Cold Spring Harb Protoc (2):3944

Sedlak M, Ho NW (2004) Characterization of the effectiveness of hexose transporters for transporting xylose during glucose and xylose cofermentation by a recombinant Saccharomyces yeast. Yeast 21(8):671-684

Shiba Y, Paradise EM, Kirby J, Ro DK, Keasling JD (2007) Engineering of the pyruvate dehydrogenase bypass in Saccharomyces cerevisiae for high-level production of isoprenoids. Metab Eng 9(2):160-168

Silversand C, Haux C (1997) Improved high-performance liquid chromatographic method for the separation and quantification of lipid classes: application to fish lipids. J Chromatogr B Biomed Sci Appl 703(1-2):7-14

Starai VJ, Gardner JG, Escalante-Semerena JC (2005) Residue Leu-641 of acetyl-CoA synthetase is critical for the acetylation of residue Lys-609 by the protein acetyltransferase enzyme of Salmonella enterica. J Biol Chem 280(28):26200-26205

Steinbuchel A, Hustede E, Liebergesell M, Pieper U, Timm A, Valentin H (1993) Molecular basis for biosynthesis and accumulation of polyhydroxyalkanoic acids in bacteria. FEMS Microbiol Rev 10(3-4):347-350

Steinbüchel A (2001) Perspectives for biotechnological production and utilization of biopolymers: Metabolic engineering of polyhydroxyalkanoate biosynthesis pathways as a successful example. Macromol Biosci 1(1):1-24

Steinbüchel A, Hein S (2001) Biochemical and molecular basis of microbial synthesis of polyhydroxyalkanoates in microorganisms. In: Babel W, Steinbüchel A (eds) Adv BiochemEng/Biotechnol, vol 71. Springer Berlin, Heidelberg, pp 81-123

Suzuki Y, Kurano M, Arai Y, Nakashita H, Doi Y, Usami R, Horikoshi K, Yamaguchi I (2002) Enzyme Inhibitors to increase poly-3-hydroxybutyrate production by transgenic tobacco. Biosci Biotechnol Biochem 66(12):2537-2542

Trotsenko YA, Belova LL (2000) Biosynthesis of poly(3-Hydroxybutyrate) and poly (3-hydroxybutyrate-co-3-hydroxyvalerate) and its regulation in bacteria. Microbiol 69(6):635-645

Tyo KE, Zhou H, Stephanopoulos GN (2006) High-throughput screen for poly-3hydroxybutyrate in Escherichia coli and Synechocystis sp. Strain PCC6803. Appl Environ Microbiol 72(5):3412-3417

Uchino K, Saito T (2006) Thiolysis of poly(3-hydroxybutyrate) with polyhydroxyalkanoate synthase from Ralstonia eutropha. J Biochem 139(3):615-621

van Wegen RJ, Lee S-Y, Middelberg APJ (2001) Metabolic and kinetic analysis of poly(3-hydroxybutyrate) production by recombinant Escherichia coli. Biotechnol Bioeng 74(1):70-81

Zhang B, Carlson R, Srienc F (2006) Engineering the monomer composition of polyhydroxyalkanoates synthesized in Saccharomyces cerevisiae. Appl Environ Microbiol 72(1):536-543

doi:10.1186/2191-0855-2-52

Cite this article as: Kocharin et al.: Engineering of acetyl-CoA metabolism for the improved production of polyhydroxybutyrate in Saccharomyces cerevisiae. AMB Express 2012 2:52.

\section{Submit your manuscript to a SpringerOpen ${ }^{\circ}$ journal and benefit from:}

- Convenient online submission

$\checkmark$ Rigorous peer review

- Immediate publication on acceptance

- Open access: articles freely available online

- High visibility within the field

- Retaining the copyright to your article

Submit your next manuscript at $>$ springeropen.com 